\title{
A GPS based fawn saving system using relative distance and angle determination
}

\author{
A. Ascher, M. Eberhardt, M. Lehner, and E. Biebl \\ Associate Professorship of Microwave Engineering, Technical University of Munich, Munich, Germany \\ Correspondence to: A. Ascher (alois.ascher@tum.de)
}

Received: 17 December 2015 - Revised: 26 February 2016 - Accepted: 28 February 2016 - Published: 28 September 2016

\begin{abstract}
Active UHF RFID systems are often used for identifying, tracking and locating objects. In the present publication a GPS- based localization system for saving fawns during pasture mowing was introduced and tested. Fawns were first found by a UAV before mowing began. They were then tagged with small active RFID transponders, and an appropriate reader was installed on a mowing machine. Conventional direction-of-arrival approaches require a large antenna array with multiple elements and a corresponding coherent receiver, which introduces a large degree of complexity on the reader-side. Instead, our transponders were equipped with a small GPS module, allowing a transponder to determine its own position on request from the reader. A UHF link was used to transmit the location to a machine- mounted reader, where a second GPS receiver was installed. Using information from this second position and a machine- mounted magnetometer for determining the relative north direction of a vehicle, relative distance, and angle between GPS receivers can be calculated. The accuracy and reliability of this novel method were tested under realistic operating conditions, considering critical factors such as the height of grass, the lying position of a fawn, humidity and geographical area.
\end{abstract}

\section{Introduction}

The main period for fawns to be born is in the spring, between the end of April and middle of June (Rieck, 1955). When giving birth, does seek calm and protected areas on the edges of woods with high grass as a visual cover, to protect newborn fawns against natural threats. Unfortunately, this birth period coincides with the first cut of the pasture when farmers start mowing for animal forage. To make matters worse, fawns instinctively lie flat and silent on the ground when danger approaches. This strategy works well against their natural predators such as foxes or wolves, but leads to a large number of fawns being killed by mowing machines running over them. In recent years, numerous research projects have been conducted in an attempt to tackle this problem. Systems based on infrared or radar detection were developed by Fackelmeier and Biebl (2009), but these systems generated an excessive rate of false positives and required delicate technologies that were impractical in the rough agricultural working environment. Traditionally, farmers in a particular area begin mowing at the same time, which means that the large field area cannot be searched on foot immediately before mowing. To address this problem a four step process has been introduced to decouple the mowing from the searching procedure. The four defined steps are finding, marking, recovering and rescuing. A few days before mowing begins, fawns are detected using a UAV (Israel, 2011) or other suitable technology. If a fawn is found, it is marked with a small active RFID transponder, making it is easy to later detect the fawn using a corresponding reader, mounted on a mowing machine. This solves the problem of false-positive alarms. A conventional direction of arrival system fuses a coherent receiver and a corresponding antenna array (Eberhardt et al., 2015 b), which leads to high complexity on the reader- side. In an alternative approach, GPS modules have been used in the transponder and reader. Using positional information of the transponder- and reader GPS modules and the additional correction values from a machine mounted magnetometer to determine the orientation of the vehicle, it is possible to calculate the relative distance and angle between the GPS receivers. Numerous animal-tracking systems have used RFID and GPS for example, (Zviedris et al., 2010). However these have been developed for the narrow field of animal research, and are not suitable for widespread applications. 


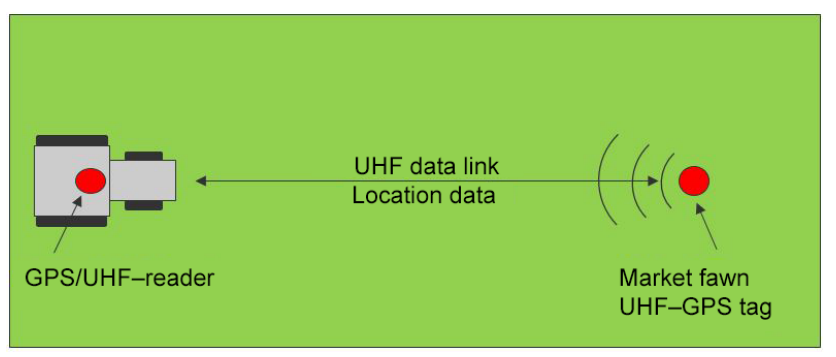

Figure 1. Detection of a fawn using a UHF data link and localization information.

\section{System description}

The system developed in this research avoided the use of conventional direction-of-arrival techniques to allow it to be integrated with different types of mowing machine. Systems requiring wide antenna structures and large receiver setups connected to them make this challenging, because of the different shapes and sizes of mowing machines from different manufacturers. A further goal was that the system should be able to communicate with the agricultural machine using the standard ISOBUS protocol. The envisaged functionality is shown in Fig. 1. The transponder on a marked fawn would reveal its own position on request from the reader. After a valid set of coordinates was identified, this information would be send to the machine mounted reader, where further data processing is performed.

The hardware comprised a transponder for tagging the fawn and a reader integrated into the agricultural machine. A number of prerequisites have to be fulfilled.

The transponder presented in Ascher et al. (2015) had to comply a small form factor of less than $30 \mathrm{~mm} \times 50 \mathrm{~mm}$. A transponder larger than this, cannot be attached to the fawn without disturbing its natural behavior. The GPS module had to have a lifetime of at least four weeks, over which period it had to perform a minimum of three GPS fixes. The time taken to make valid GPS coordinates available had to be less than $60 \mathrm{~s}$, and the communication range of the UHF data link had to be at least $200 \mathrm{~m}$ to guarantee that the mowing machine could be stopped in time. The realized transponder is shown in Fig. 2, both in close-up and attached to a fawn. We also wanted to ensure that the transponder had no major environmental impacts. Because batteries need to be used in active UHF transponders, these have to be chosen while taking account of their flammability and environmental sustainability. After comparing batteries based on different technologies, zinc air $(\mathrm{ZnO})$ primary cells were selected as the most suitable, as they are capable of providing a peak current of over $100 \mathrm{~mA}$ which is drawn from the GPS module during acquisition, are nonflammable, and produce comparatively safe chemical reaction products.

The transponder comprised a high efficiency GPS module (Origin), a CC1110 System on a Chip (SoC) with an inte-

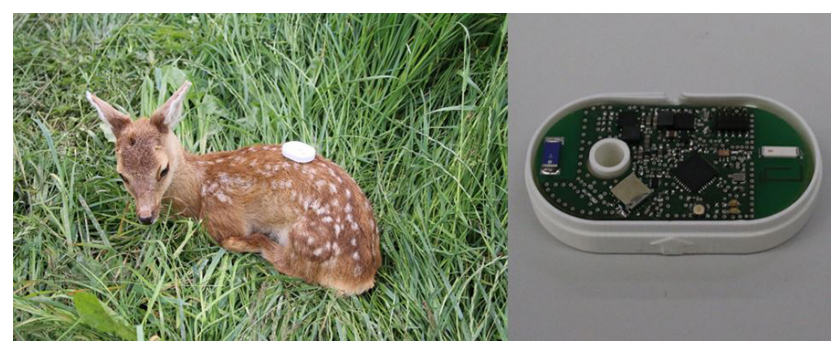

Figure 2. Marked fawn dummy and realized GPS- UHFtransponder.

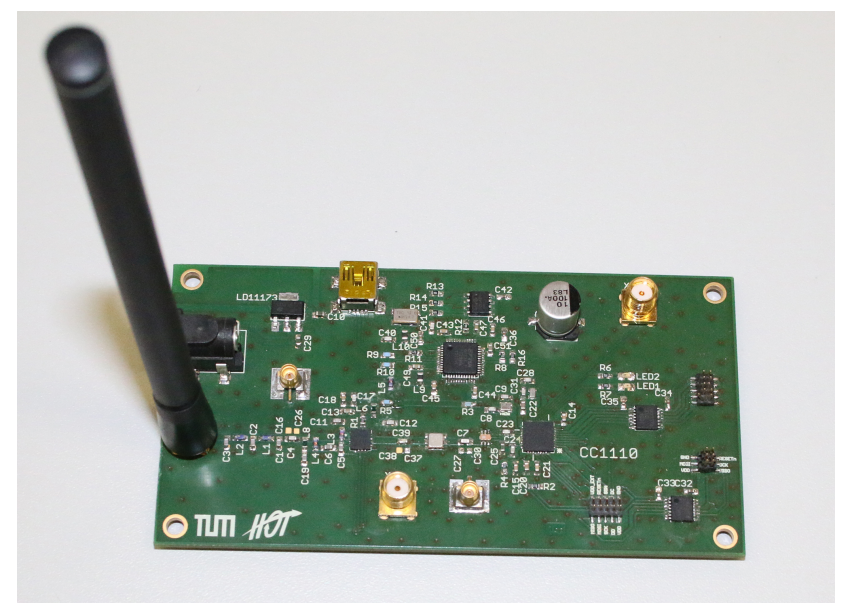

Figure 3. The machine mounted reader.

grated UHF frontend for the data link, chip antennas for the UHF- and GPS L1 band to meet the size constraints, and four stacked $\mathrm{ZnO}$ batteries at the back of the transponder as a power supply.

For integration with the mowing machine, the comparably small reader board shown in Fig. 3 was developed. The reader itself used the same $\mathrm{SoC}$ as the transponder, but includes an additional variable gain amplifier (VGA) to enhance both the receiver sensitivity and the transmitting power. For communication with the mowing machine, an FTDI based USB interface was available. The board had a $12 \mathrm{~V}$ operating voltage, matching the standard on-board power supply of agricultural machinery. For localization, an additional GPS module was mounted on the roof of the machine and connected to the reader board using UART. It is possible to replace this supplementary GPS module with the high precision DGPS which is often implemented in agricultural machines for lane detection. The system was therefore straightforward to integrate into the existing communication protocols of the mowing machines, whilst providing high precision localization.

Figure 4 shows the flowchart of the system. The system starts in Status 1, at which point the transponder is not within range of the reader. In this status, the transponder switches its power mode to energy saving (TI, 2013). The reader with 


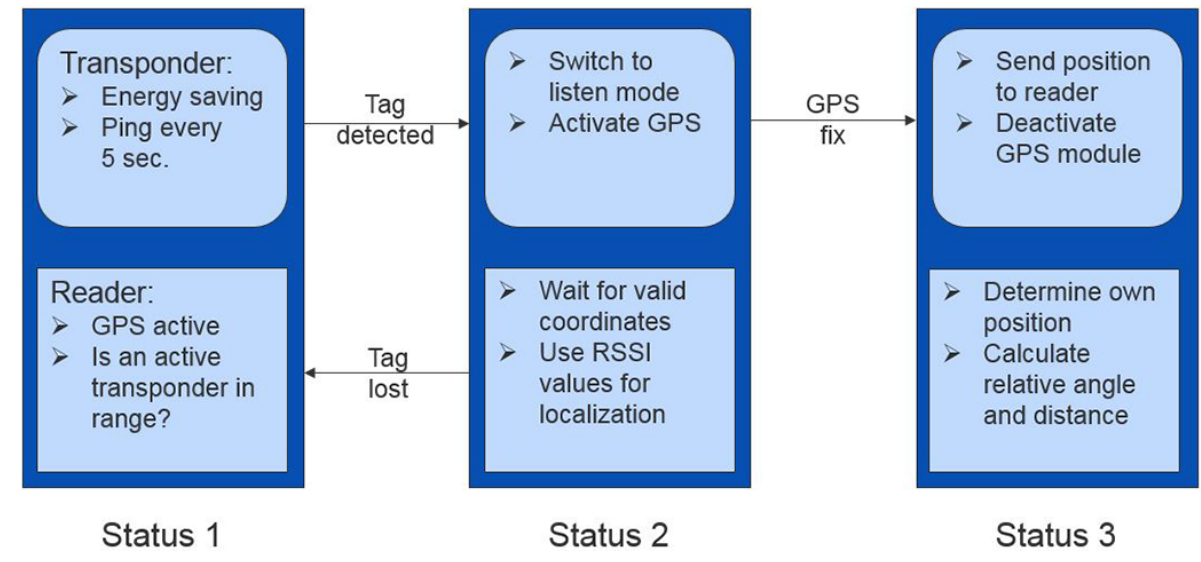

Figure 4. System flowchart.

the vehicle-mounted GPS continuously updates its position and seeks any active transponder is in range sending its ID. This is the main operating state of the system, in which the transponder draws an average current of $60 \mu \mathrm{A}$. Status 2 is the phase in which a transponder is detected and communicates with the reader, but no valid coordinates are yet available. The transponder and the reader change roles, performing as a dynamic master-slave pairing. In response to the commands of the reader, the transponder activates its integrated GPS module. The reader then requests a valid transponder position. If no position can be determined, a simple but very inaccurate RSS based localization is performed to protect the fawn pending acquisition. If a GPS fix is achieved, the system moves to Status 3, in which the GPS module of the transponder is switched off and the coordinates are sent to the reader using the UHF link. The vehicle-mounted reader determines its own position by reading out the coordinates from its integrated GPS via ISOBUS. Using these two pieces of information, it is possible to calculate the relative distance and angle between fawn and mowing machine. To calculate the relative angle between the reader and the transponder, the orientation of the machine must be known. If the tractor is stationary, because the mower is being prepared ahead of the mowing process, the direction cannot be calculated from changes in the GPS trajectories. The same problem arises when a handheld device like that presented in Eberhardt et al. (2015a) is used separately from the machine. In this case, an additional machine-mounted magnetometer can be used to determine the orientation even if the machine is stopped.

\section{Calculating relative distance and angle}

If both positions are available, for example using WGS84 coordinates (Misra and Enge, 2006) it is possible to calculate the relative distance and angle using the coordinate sets:

$c_{\text {tag }}=\left(\begin{array}{c}l_{\text {lat }} \text { tag } \\ \operatorname{lon}_{\text {tag }}\end{array}\right) \quad c_{\text {reader }}=\left(\begin{array}{c}l_{\text {at }} \text { reader } \\ \text { lon }_{\text {reader }}\end{array}\right)$
First, the distance between the GPS positions is calculated. The Haversine formula (Sinnott, 1984) supplies a good approximation across short distances and ignoring varying altitude. The coordinates of the transponder and the reader are fed into the Haversine formula, and the distance between the tag and reader, $\boldsymbol{d}_{\text {tag-reader }}$ is derived. This length is then projected onto the $y$ axis as follows:

$\boldsymbol{v}_{\text {tag-reader }}=\left(\begin{array}{c}0 \\ d_{\text {tag-reader }}\end{array}\right)$

By using a correction angle $\epsilon$, obtained from the moving trajectories or the magnetometer the projected distance is rotated using a rotational matrix $\mathbf{R}$ :

$\mathbf{R}=\left[\begin{array}{cc}\cos (\epsilon) & -\sin (\epsilon) \\ \sin (\epsilon) & \cos (\epsilon)\end{array}\right]$

$\boldsymbol{v}_{\text {rotated }}=\boldsymbol{v}_{\text {tag-reader }} \cdot \mathbf{R}$

The difference of the coordinate sets in latitude and longitude is then used to calculate the relative angle:

$\boldsymbol{v}_{\text {diff }}=\left(\begin{array}{c}l_{\text {atead }}-1 \mathrm{lat}_{\mathrm{tag}} \\ \operatorname{lon}_{\mathrm{read}}-\operatorname{lon}_{\mathrm{tag}}\end{array}\right)$

It is now possible to calculate the relative angle using the four quadrant arctangent atan2 (Jones, 1991), where atan2 is denoted as an arctangent with two arguments instead of one to obtain the sign of the inputs and decide the appropriate quadrant of the output angle $\alpha$.

$$
\begin{array}{r}
\alpha=\left(\operatorname{atan} 2\left(\boldsymbol{v}_{\text {rotated }}(1,2), \boldsymbol{v}_{\text {rotated }}(1,1)\right)\right. \\
\left.-\operatorname{atan} 2\left(\boldsymbol{v}_{\text {diff }}(1,2), \boldsymbol{v}_{\text {diff }}(1,1)\right)\right) \cdot \frac{180}{\pi}
\end{array}
$$

Using this equation for relative angle $\alpha$ and the distance $\boldsymbol{d}_{\text {tag-reader }}$ from the Haversine formula, it is possible to visualize the context of two arbitrary sets of coordinates. 


\section{Measurement results}

To demonstrate the system, trails were performed under operational conditions in open grassland. The reader was mounted on the roof of a tractor, and the GPS transponder was placed in waist-high grass. To examine the performance, the measurements were taken at different locations in the environment taking account of geographical conditions, including different heights and partial coverage of the sky by a hill. Because an animal test permission would have to be gained to attach the transponder on live fawns in the experiment, a model of a fawn filled with material to simulate the electromagnetic behavior of muscle tissue (Hartsgrove et al., 1987) was used. As a result, a test with real fawns was not mandatory. The transponder was mounted horizontally during all measurements, because fawns are known to prefer a lying position, and the transponder will typically be attached to the fawn's back or neck (Fig. 2). Thereby the localization errors for vertical mounting are irrelevant for the desired application. To determine the performance of the system, measurements were performed using an immobile mowing machine. The transponder integrated GPS was therefore always operating under cold-start conditions, which implicates that no ephermis data were available. The coordinates at the transponder were sent to the reader using the UHF data link, stored with the self-monitored position of the reader, and analyzed in real time. The true distance between the reader and transponder was $208 \mathrm{~m}$, at a relative angle of $33^{\circ}$. At least seven satellites were accessible by the reader and the transponder during all measurements for position calculation, with a mean satellite availability of nine. An initial measurement series shall be discussed to investigate the expected system performance. The measurement time was 18 min with an updating rate for the GPS coordinates of $1 \mathrm{~s}$. As the measurement was run under cold-start conditions, and the expected sample count was 1080 for a measurement time of $18 \mathrm{~min}$ with an update rate of $1 \mathrm{~s}$, the cold-start time was calculated from the 1039 samples achieved as follows:

$t=1080-1039=41 \mathrm{~s}$

The cold-start time of $41 \mathrm{~s}$ met the transponder requirements. Figure 5 shows the recorded positions of the reader against the mean circular error probability (CEP) within $50 \%$ of the measured points are lying. With a CEP of $0.5 \mathrm{~m}$, and a twice distance root mean square (2DRMS) in which $95 \%$ of the points were within $1.38 \mathrm{~m}$, the precision of the readerside module was relatively high.

Figure 6 shows the same information but for the transponder-side GPS. With a CEP of $0.41 \mathrm{~m}$ and a 2DRMS of $1.68 \mathrm{~m}$, the precision was comparable to that of the readerside module. The coarser grid used on the transponder-side reflected the poorer horizontal resolution provided by the Origin module of the transponder, compared to the Maestro module used in the vehicular system.

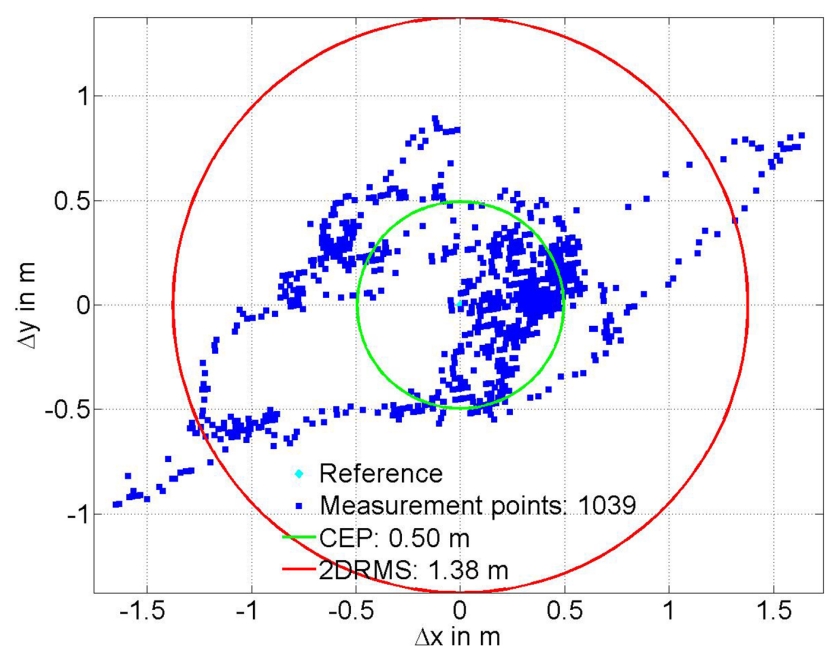

Figure 5. Recorded positions at the reader.

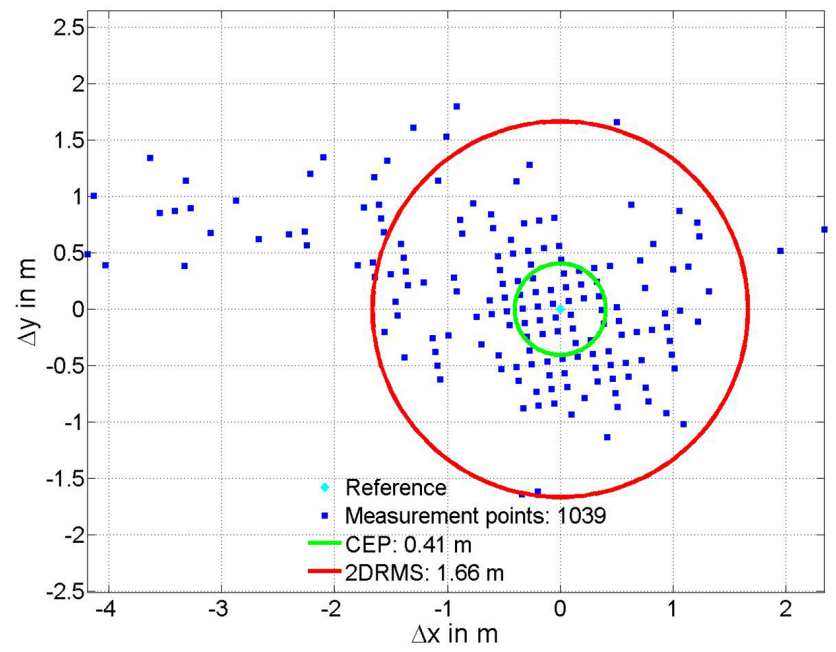

Figure 6. Recorded positions at the transponder.

Using the measured coordinate pairs and the formulas presented in Sect. 3, it was possible to calculate the relative angle and distance to the fawn. The driver of the mowing machine was given a user-friendly interface, as shown in Fig. 7. This was designed, to combine a continuously updated satellite map showing an overview of the terrain and the remaining distance to the fawn, and a stylized compass to show the relative direction of the vehicle's movement. To analyze the system performance, we examined the deviation of the calculated distance and angle. The shown results are thereby comparable to the further measurement series. As can be seen in Fig. 8, the deviation from the true relative angle of $33^{\circ}$ and distance of $208 \mathrm{~m}$, were small. This leads to a mean angle of $32.075^{\circ}$ and a maximum error of $2.2^{\circ}$ without any smoothing or filtering of the data. The calculated distance was equally accurate, with a mean ascertained distance of $209.4 \mathrm{~m}$ and a maximum error of $3.8 \mathrm{~m}$, equal to an error of less than $2 \%$. 


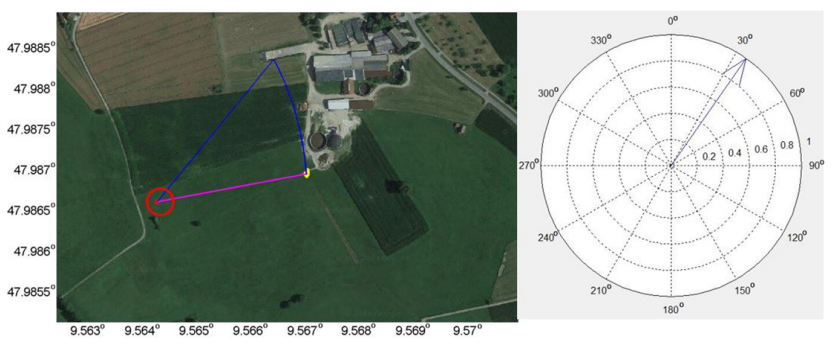

Figure 7. Interface provided to the driver of the mowing machine. The red circle and dot on the map denote the machine position, with the blue line pointing in the direction of movement. The magenta line points towards the fawn's location, represented by the yellow dots. The compass shows the relative direction to the fawn with relation to the movement trajectory.
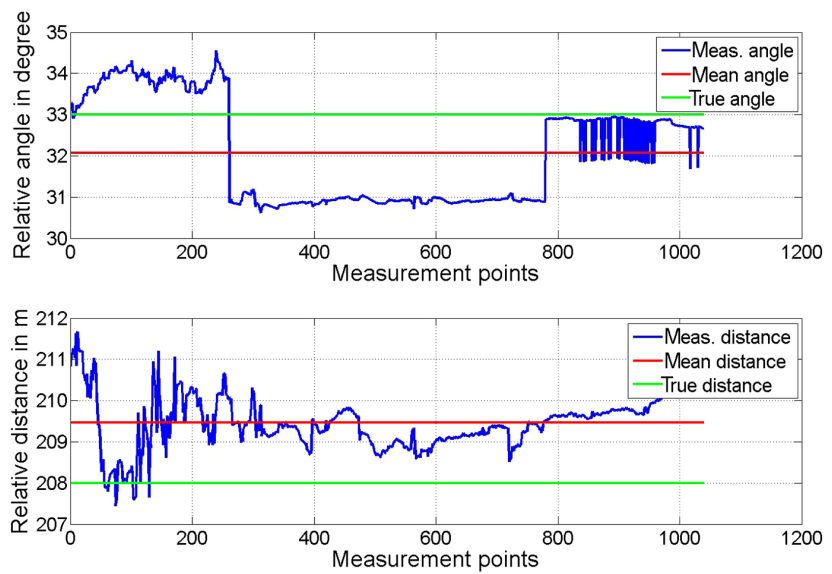

Figure 8. Evaluation of the angle and distance deviation over time.

Finally, the worst case estimation of the angular error as a function of the distance was made. The calculated errors were compared with regard to the number of points in the underlying measurement series. The maximum error was based on the single coordinate pair with the largest angular error. The 50 and $90 \%$ errors are with regard to the maximum error observing the worst measured angle, taking account of the best fitting 50 or $90 \%$ of the coordinate pairs. All coordinate sets were used in the calculation of the mean error. The extrapolated distance dependent angular errors are shown in Fig. 9.

As the distance between the reader and the transponder became shorter, the results became increasingly inaccurate. That could create problems when approaching the fawn during the recovery process. A possible solution would be to combine the GPS based localization used over longer distances with a small handheld single point locator based on the conventional direction-of-arrival technique presented in (Eberhardt et al., 2015a). This would supplement the presented GPS based localization over the last few meters, improving the success rate and speed of retrieval.

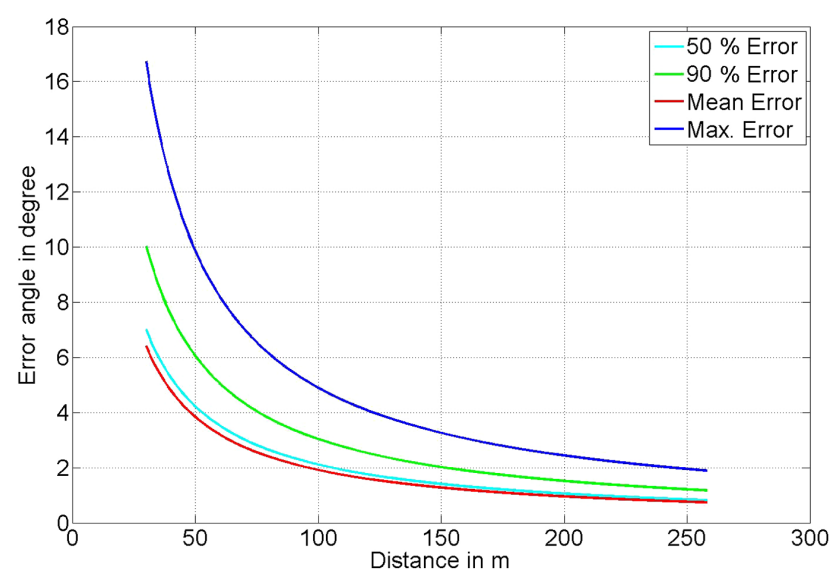

Figure 9. Relative angle error as function of distance due to GPS localization precision.

\section{Conclusions}

A system for identifying the location of fawns during pasture mowing was developed. The system, comprised a machinemounted reader and a transponder attached to the fawn. In place of conventional direction-of-arrival approaches, a GPS based localization system was used for determining the relative angle and distance. An additional GPS receiver was integrated with the active UHF transponder attached to the fawn and the vehicle-mounted reader. The transponder coordinates were sent to the reader unit via a UHF data link with an updating rate of $1 \mathrm{~s}$. Using the provided coordinates and a magnetometer on the reader-side, it was shown to be possible to calculate the relative angle and distance. Measurements were performed under realistic operating conditions to verify the practicality of the overall system. It was shown that the distance and angle could be very precisely estimated when the transponder and reader were separated by more than $100 \mathrm{~m}$. To avoid large errors as the separation between the transponder and reader becomes smaller, a combined approach is suggested, in which direction finding over long distances is performed using the GPS based system. If the mowing machine is closer than $20 \mathrm{~m}$ the machine should stop and a conventional handheld direction-of-arrival system can guide the driver to the fawn on foot. The influence of mechanical movements on the machine-mounted reader should also be evaluated to establish the final design and optimum vehicular mounting position. 
Acknowledgements. The project is supported by funds of the Federal Ministry of Food, Agriculture and Consumer Protection (BMELV) based on a decision of the Parliament of the Federal Republic of Germany via the Federal Office for Agriculture and Food (BLE) under the innovation support program.

This work was supported by the German Research

Foundation (DFG) and the Technische Universität

München within the funding programme

Open Access Publishing.

Edited by: J. Anders

Reviewed by: two anonymous referees

\section{References}

Ascher, A., Eberhardt, M., Lehner, M., Lippert, B., and Biebl, E.: A small UHF-RFID transponder with integrated GPS for localization applications, International EURASIP Workshop on RFID Technology (EURFID), Rosenheim, Germany, 22-23 October 2015, 132-139, 2015.

Eberhardt, M., Ascher, A., Lehner, M., and Biebl, E.: Array Manifold Manipulation for Short Distance DOA Estimation with a Handheld Device, IEEE European Conference on Smart Objects, Systems and Technologies (SmartSysTech), Aachen, Germany, 16-17 June 2015, 1-7, 2015a.
Eberhardt, M., Lehner, M., Ascher, A., Allwang, M., and Biebl, E. M.: An active UHF RFID localization system for fawn saving, Adv. Radio Sci., 13, 87-94, doi:10.5194/ars-13-87-2015, 2015 b.

Fackelmeier, A. and Biebl, E.: A multistatic radar array for detecting wild animals during pasture mowing, EuRAD 2009 - European Radar Conference, Rome, Italy, 30 September-2 October 2009, 477-480, 2009.

Hartsgrove, G., Kraszewski, A., and Surowiec, A.: Simulated Biological Materials for Electromagnetic Radiation Absorption Studies, Bioelectromagnetics, 8, 29-36, doi:10.1002/bem.2250080105, 1987

Israel, M.: A UAV-based Roe Deer Fawn Detection System, Proceedings of International Conference on Unmanned Aerical Vehicle in Geomatics (UAV-g), Zurich, Switzerland, 14-16 September 2011, 1-5, 2011.

Jones, R. S.: The C Programmers Companion, 1st Edn., Silicon Press, New York, USA, 151 pp., 1991.

Misra, P. and Enge, P.: Global Positioning System. Signals, Measurements, and Performance, 2nd Edn., Ganga-Jamuna Press, New York, USA, 590 pp., 2006.

Rieck, W.: Die Setzzeit bei Reh-, Rot- und Damwild in Mitteleuropa, Zeitschrift für Jagdwissenschaft, 1, 69-75, 1955.

Sinnott, R. W.: Virtues of the Haversine, Sky and Telescope, 68, p. 159, 1984.

TI, T. I.: CC1110Fx/CC1111Fx Low-Power SoC (System-on-Chip) with MCU, Memory, Sub-1 GHz RF Transceiver, and USB Controller data sheet, 2013.

Zviedris, R., Elsts, A., Strazdins, G., Mednis, A., and Selavo, L.: LynxNet: Wild Animal Monitoring Using Sensor Networks, 4th International Workshop Real-World Wireless Sensor Networks, Colombo, Sri Lanka, 16-17 December 2010, 170-173, 2010. 